\title{
Análisis de contenidos de la lista de correo-e lweTel (2001-2007)
}

\author{
Por José-Antonio Ontalba-Ruipérez
}

\begin{abstract}
Resumen: Se estudia la evolución de la lista de correo electrónico IweTel, y el uso que los suscriptores han hecho de ella, a partir del análisis de contenido de los mensajes que fueron enviados entre 2001 y 2007. Del estudio se desprende que se trata de una lista utilizada fundamentalmente para la comunicación profesional y el intercambio de información y sobre todo por la comunidad universitaria, aunque adolece de una falta notoria de implicación activa por parte de sus suscriptores. Por otro lado, ha mejorado la gestión de la lista e incluso los debates desarrollados en los últimos años.
\end{abstract}

Palabras clave: Lista de distribución, Lista de discusión, Lista de correo electrónico, Comunidades virtuales, IweTel, Listserv, Análisis de contenido, Comunicación profesional, Comunicación científica.

Title: Content analysis of the IweTel e-mailing list (2001-2007)

Abstract: The evolution of the mailing list IweTel is presented, including a description of its evolution and how it has been used by its subscribers, by analysing the content of the messages sent from 2001 to 2007. The study shows that the list is used mainly for professional communication and information exchange and, overall, by the academic community. However, a lack of active participation by its members is also revealed. On the other hand, list management has improved in recent years, and so has the quality of the debates.

Keywords: Distribution list, Discussion list, Mailing list, Virtual Communities, IweTel, Listserv, Content analysis, Professional communication, Scientific communication.

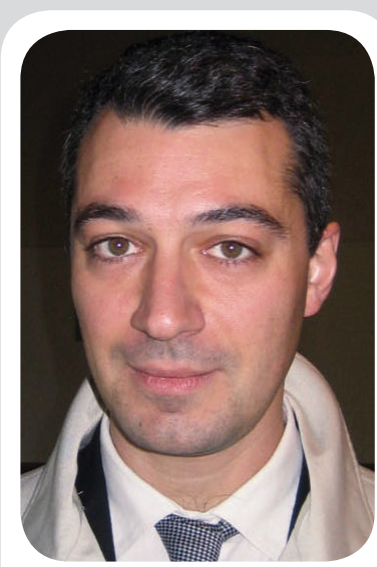

José-Antonio Ontalba-Ruipérez, licenciado en geografía e historia, licenciado y doctor en documentación, es profesor de los estudios de documentación en la Universidad Politécnica de Valencia.

Ontalba-Ruipérez, José-Antonio. Análisis de contenido de la lista de correo electrónico IweTel (2001-2007). El profesional de la información, 2009, enero-febrero, v. 18, n. 1, pp. 50-61.

DOI: 10.3145/epi.2009.ene.07

\section{Introducción}

Tras su expansión durante la década de los 80 y, sobre todo, la de los 90, las listas de correo electrónico, también conocidas como listas de distribución o foros de discusión, se han asentado como un importante canal de comunicación profesional y científica.

No sólo permiten mantener informados a sus suscriptores sobre la actualidad en su ámbito de acción (noticias sobre actividades de personas, instituciones, programas, proyectos...), sino que generan espacios comunes para la vertebración social de los grupos (cooperar, compartir recursos, consultar dudas e intercambiar experiencias, emitir y confrontar opiniones...) (Burton, 1994; Robinson, 1996). Se las consideró como el primer paso en el camino hacia las comunidades virtuales (Burnett, 2000; Ellis, et al. 2004), pero, en los últimos años, y en paralelo al desarrollo de los servicios de la Web social que incluyen herramientas con más y mejores prestaciones para la comunicación personal, se están viendo sometidas a la evolución y adaptación a una comunidad profesional con una ma- yor especialización disciplinar que está cambiando sus hábitos comunicativos.

La relevancia que las listas de correo han adquirido como medios de información profesional, y su progresiva implantación en los más diversos sectores de actividad, ha generado una abundante bibliografía. Desde finales de los 80 menudean los artículos que describen las características de las listas de correo electrónico y enumeran sus aplicaciones y ventajas (Hartland; "New Listserv for (...)"). Sobre todo entre 1993 y 1998, se multiplican los textos, en muchas ocasiones de una extensión mínima, que anuncian la aparición de nuevas listas o cambios en éstas, recopilan ejemplos temáticamente, entrevistan a los responsables, etc. (García-Testal; Gilas; Merlo, Sorli). Las áreas profesionales en las que más se ha publicado sobre listas de distribución son Medicina (Rodríguez-Recio, SendraPortero; Schoch, Shooshan), Documentación (Merlo, Sorli; Ontalba-Ruipérez), Ciencias de la educación (Althaus), Psicología (Tichon, Shapiro) e Informática (Moreale, Watt). 
Desde mediados de los 90 empiezan a proliferar trabajos empíricos que toman como objeto de estudio las propias listas de correo. Al margen de los estudios de casos (Klinger, Ray) y de los trabajos descriptivos (Mac Lennan), se han ido abriendo varias líneas de investigación:

- una de ellas se centra en la evaluación de la calidad de las listas y en la propuesta de parámetros a tal fin (Feliu; Pedersen; Kollock);

- otra examina el comportamiento de los usuarios, las relaciones entre éstos, el papel de los moderadores $y$, en general, la generación de conocimiento en estas herramientas de comunicación (Berge; Collins);

- y otra más se basa en el análisis de contenido (Pubyac, Serialst, Radiología, Neurología, Asis-L, etc.), que lleva en algunos casos a estudios bibliométricos sobre la productividad de las listas (Bar-Ilan;

\section{Kuperman).}

IweTel, creada en 1993 en la entonces empresa Spritel (hoy Sarenet) por Tomàs Baiget, que buscaba un canal de información profesional de actualización diaria que complementara a la revista Information world en español (IWE), se ha convertido en un canal de información imprescindible en el ámbito de la $\mathrm{Bi}$ blioteconomía y Documentación españolas y en uno de los foros de referencia en el ámbito castellanohablante. Ya fue objeto de estudio en un artículo en el que se analizó el contenido de los mensajes de la lista para los años 1998 a 2000, los primeros en conservarse (Ontalba-Ruipérez, 2002).

El presente trabajo es, por tanto, una actualización de aquél y se plantea como fin analizar la evolución de la lista desde 2001 hasta 2007 con los siguientes objetivos específicos:

- Describir la evolución del número de suscriptores y mensajes de IweTel.

- Describir la evolución del número de autores, su procedencia y productividad.

- Analizar el grado de participación e identificar los principales autores.

- Caracterizar la tipología de mensajes remitidos.

- Analizar los contenidos y los autores de los debates de la lista.

\section{Material y métodos}

Análisis de contenido descriptivo longitudinal retrospectivo de los 17.475 mensajes remitidos entre 2001 y 2007, que se han descargado de los archivos de IweTel (y que se publican en abierto desde el 8 de mayo de 2006):

\section{http://listserv.rediris.es/archives/iwetel.html}

Este universo se emplea para estudiar tres variables:

1. Evolución temporal: número de mensajes por año, mes y día, además del número de suscriptores.

2. Autoría: género, procedencia geográfica y filiación profesional de quienes remiten los mensajes, fijándose las pautas de productividad de la lista.

3. Contenido: análisis de los debates de la lista, indicando los autores con impacto.

En esta tercera variable se establecerá también una clasificación temática de los mensajes. Habida cuenta que ello requiere del análisis del contenido de éstos, sólo en este caso se ha optado por utilizar una muestra de 2.170 mensajes, elegida por un procedimiento de muestreo por conglomerados, presentando un margen de confianza de $99,7 \%$ y un error estándar de $\pm 3 \%$. En concreto, se han seleccionado los 155 primeros mensajes de los meses de febrero y octubre (los más productivos) de cada uno de los años. Se han elegido los mensajes en grupos secuenciales dentro de cada mes para poder identificar y seguir los debates desarrollados en la lista.

Si bien el volumen del universo obliga a que su análisis se haga a partir de los datos aparecidos en la cabecera de los mensajes (ya que así se facilita su tratamiento automatizado), en el caso de la muestra se trabaja con el cuerpo de los mensajes, puesto que es un volumen de información asequible.

En lo referente a la variable "origen geográfico", los valores se analizan, en cuanto a España, a nivel de comunidad autónoma y, respecto al resto de países, a nivel nacional.

En cuanto al ámbito profesional se utilizan cuatro categorías:

- Universidad: mensajes remitidos tanto por profesores universitarios, como por bibliotecarios de universidades o estudiantes.

- Administración: mensajes remitidos por personas que trabajan en algún área de la Administración pública (museos, bibliotecas, archivos, hospitales, centros culturales, organismos públicos de investigación (OPIs), embajadas, ayuntamientos, gobiernos autonómicos, nacionales, etc.).

- Empresa: mensajes originados desde alguna organización privada con ánimo de lucro.

- Entidades: organismos no pertenecientes a la Administración Pública ni con ánimo de lucro (asociaciones, colegios profesionales, fundaciones, plataformas, ONGs, etc.).

Finalmente, para describir la temática y tipo de los mensajes se utilizan las categorías planteadas por Ontalba-Ruipérez (2002): 


\section{Intercambio de información}

- Petición: solicitud de información de todo tipo; de contacto con ciertos profesionales; sobre convocatorias, cursos, etc.; de experiencias previas; sobre concepción, desarrollo y gestión de un servicio; incluye, además, encuestas y estudios.

- Respuesta: contestación directa a alguna petición de información concreta.

- Difusión: recopilación y envío a la lista de la información recibida en respuesta a una solicitud hecha por el autor de la compilación.

\section{Anuncios}

- Curso: anuncios de cursos y ofertas formativas regladas o no.

- Evento: divulgación de noticias relacionadas con la celebración de congresos, jornadas, conferencias, simposios, presentaciones, exposiciones, y otro tipo de actividades profesionales; no sólo anuncio del evento, sino también novedades (cambios) en el desarrollo de éste y otras noticias.

- Convocatoria: ofertas de trabajo, becas, ayudas a proyectos, concursos, colaboración en grupos de investigación, certificación, etc.

\section{Novedades}

- Bibliográficas: publicación de informes; borrador de guías, tesauros, publicaciones...

- Profesionales: nombramientos; ofertas, acceso gratuito a bases de datos hasta cierta fecha; divulgación de novedades profesionales. Incluye anuncios de nuevos softwares y servicios, informes sobre novedades, cambios en facultades, novedades legislativas, noticias, cambio de dirección profesional.

\section{Diversos}

Difusión de mensajes de diferente naturaleza; avisos de virus, reflexiones personales, protestas, denuncias, reivindicaciones, manifestaciones de solidaridad, posicionamientos públicos individuales o institucionales, cartas abiertas, mensajes de opinión, artículos sobre temas de interés paralelo al profesional con ánimo de provocar debate (sin conseguirlo), textos profesionales de diversa temática, chistes, divulgación de recursos, notas del grupo ThinkEPI...

http://www.thinkepi.net

\section{Duplicados}

Anuncios de oferta y canje de colecciones de duplicados y donaciones de publicaciones.

\section{Metatema}

Son los que versan sobre el uso de la lista, consejos de estilo, redacción de los temas, intervenciones de los moderadores (cambios en IweTel, aumento del número de suscriptores), etc.

\section{Debate}

Discusiones e intercambio de opiniones sobre un tema específico siempre que supere el número de cuatro mensajes.

\section{Mensajes vacíos}

Aquellos que son erróneos, inapropiados o personales, solicitudes de altas o bajas, correcciones de mensajes propios enviados anteriormente, felicitaciones, agradecimientos, etc. Actualmente, la mayoría de éstos no son aprobados por los moderadores y no se distribuyen.

\section{Repetidos}

Los enviados más de una vez a la lista.

Como sistema de recuento se ha utilizado el criterio de asignación única (una categoría temática por mensaje). Sin embargo, se encuentran problemas de interpretación propios de cualquier sistema de clasificación: hay mensajes que podrían adecuarse a más de una temática pero que, para su identificación unívoca, en este estudio se han encuadrado de manera excluyente sólo en una.

Para controlar la fiabilidad se ha aplicado un índice de concordancia (test-test) entre observadores.

Como se ha indicado antes, a la hora de analizar los mensajes sólo se han extraído los datos aparecidos en su cabecera. Esto conlleva algunos problemas de identificación de autor, de filiación profesional o de origen geográfico (por la dificultad de determinar algunos subdominios de las direcciones electrónicas).

Estos inconvenientes no se han dado en la muestra de mensajes de la que se ha analizado el contenido, puesto que el cuerpo del mensaje suelen ofrecer información suficiente como para llevar a cabo una identificación precisa.

Además, hay que tener en cuenta que algunos autores cambian de filiación profesional u origen geográfico a lo largo de los siete años. Al aplicar una única asignación por persona, se ha decidido utilizar los últimos lugares de trabajo y procedencia que aparecen.

Para el análisis de los datos se han empleado índices de frecuencias absolutas y relativas.

\section{Resultados}

\subsection{Evolución temporal}

\section{Mensajes}

Como se observa en el gráfico 1, el número de mensajes enviados a la lista no ha dejado de crecer desde 2004, alcanzando los 3.276 en 2007. 
A lo largo del período 2001-2007 se han enviado 17.475 mensajes, es decir, una media de 6,8 al día, algo superior a la media del período 1998-2000, que era de 6 .

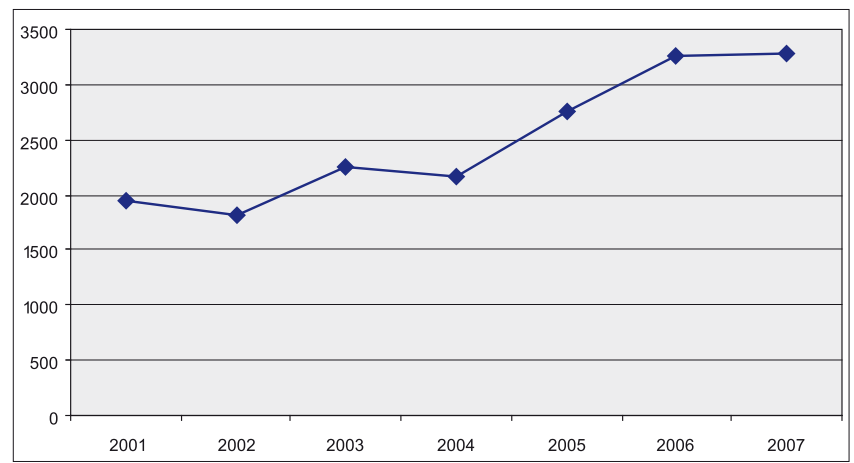

Gráfico 1. Evolución del número de mensajes por año enviados a la lista de correo electrónico IweTel (2001-2007)

Se muestra en el gráfico 2 que los meses de menor actividad siguen siendo los correspondientes a las vacaciones de Navidad, Pascua y verano (enero, abril, julio, agosto y diciembre), mientras que febrero-marzo, mayojunio, septiembre-octubre son los más productivos.

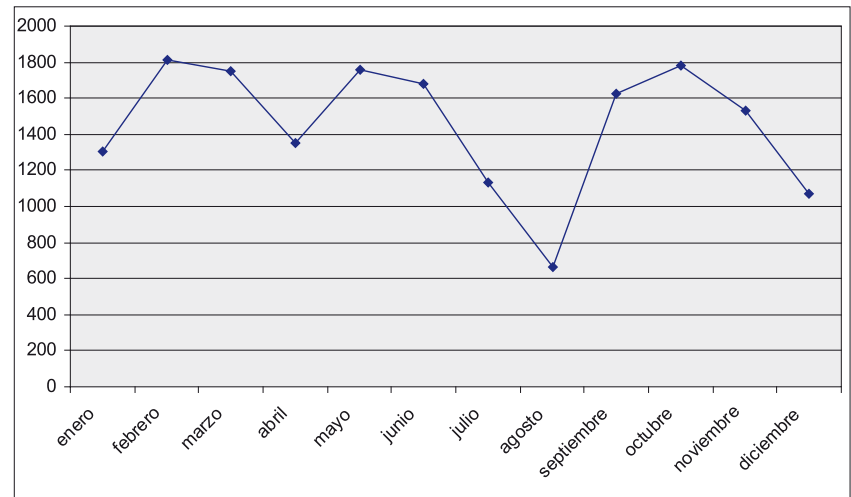

Gráfico 2. Evolución del número de mensajes por mes enviados a la lista de correo electrónico IweTel (2001-2007)

Finalmente, y como era de esperar, los días de la semana en los que se envían más mensajes son los laborables (con una distribución casi simétrica), constatándose una caída notable los sábados y domingos (gráfico 3).

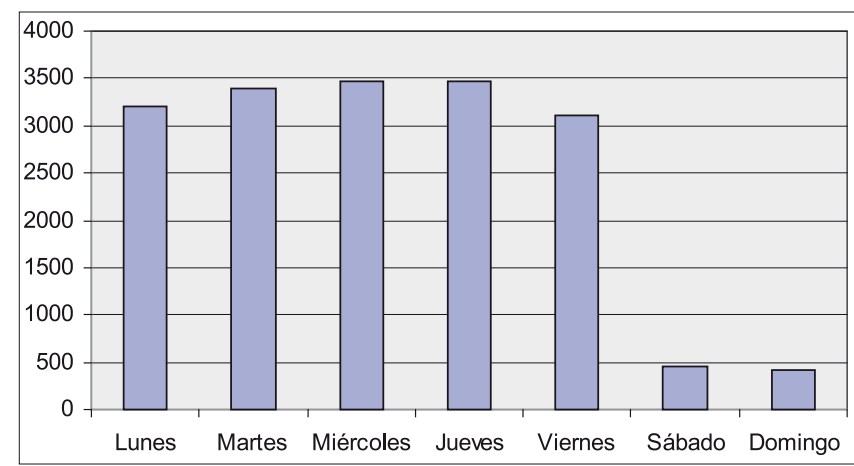

Gráfico 3. Distribución por días de la semana del número de mensajes enviados a la lista de correo electrónico IweTel (2001-2007)

\section{Suscriptores}

Siendo una lista no moderada desde su origen, el 30 de noviembre de 1999 los gestores de IweTel decidieron moderarla ante el gran número de mensajes erróneos o inadecuados que llegaban a la lista. Eso supuso un aumento sostenido de los suscriptores desde casi 3.000 hasta cerca de 5.500 en marzo de 2006 (como se puede observar en el gráfico 4). A partir de entonces el número no dejó de decrecer hasta los 4.200 a finales de 2007 , número que permanece estable durante los primeros meses de $2008^{1}$.

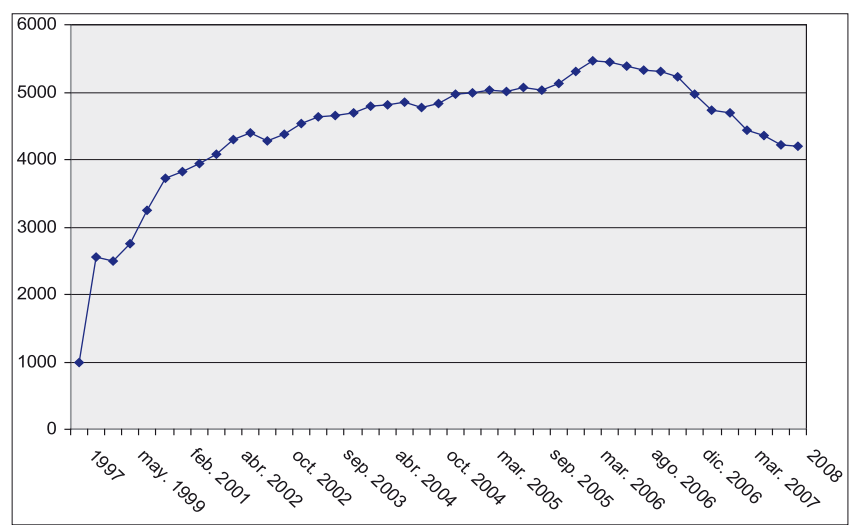

Gráfico 4. Evolución del número de suscriptores a la lista de correo electrónico IweTel (1997-2008)

A pesar de este aumento general de suscriptores, se da un descenso en la productividad de mensajes desde 2000 hasta 2004 (tabla 1). Este descenso se corrige en los años sucesivos hasta que, en 2007, el crecimiento de mensajes coincide con una bajada de suscriptores que lleva a una productividad en ese año de 0,73 mensajes por suscriptor.

\begin{tabular}{|l|c|c|c|}
\hline & Mensajes & Suscriptores & M/S \\
\hline $\mathbf{2 0 0 1}$ & 1.937 & 3.886 & $\mathbf{0 , 5 0}$ \\
\hline $\mathbf{2 0 0 2}$ & 1.825 & 4.373 & $\mathbf{0 , 4 2}$ \\
\hline $\mathbf{2 0 0 3}$ & 2.244 & 4.658 & $\mathbf{0 , 4 8}$ \\
\hline $\mathbf{2 0 0 4}$ & 2.171 & 4.838 & $\mathbf{0 , 4 5}$ \\
\hline $\mathbf{2 0 0 5}$ & 2.765 & 5.080 & $\mathbf{0 , 5 4}$ \\
\hline $\mathbf{2 0 0 6}$ & 3.257 & 5.303 & $\mathbf{0 , 6 1}$ \\
\hline $\mathbf{2 0 0 7}$ & 3.276 & 4.485 & $\mathbf{0 , 7 3}$ \\
\hline
\end{tabular}

Tabla 1. Evolución del número de mensajes y de suscriptores de la lista de correo electrónico IweTel (1998-2007)

\subsection{Autoría}

Se ha identificado un total de 2.854 autores diferentes, con una media de 6,1 mensajes por autor.

En lo que respecta a la evolución de la ratio entre autores y suscriptores, se ha estabilizado en 0,17 en los últimos años tras un descenso entre 2001 y 2003 (grá- 
fico 5). Es decir, sólo el 17\% de los suscriptores envía algún mensaje a la lista a lo largo de su pertenencia a IweTel, mientras que el $83 \%$ restante únicamente se dedica a leer lo que otros remiten.

También la distribución de los mensajes por autores parece reflejar la ley de Lotka: así, el $80 \%$ de los autores produce entre 1 y 5 mensajes ( $24 \%$ del total) [o sea que el $20 \%$ restante -autores con más de 5 mensajes- envía el 76\% de los mensajes (tabla 2)].

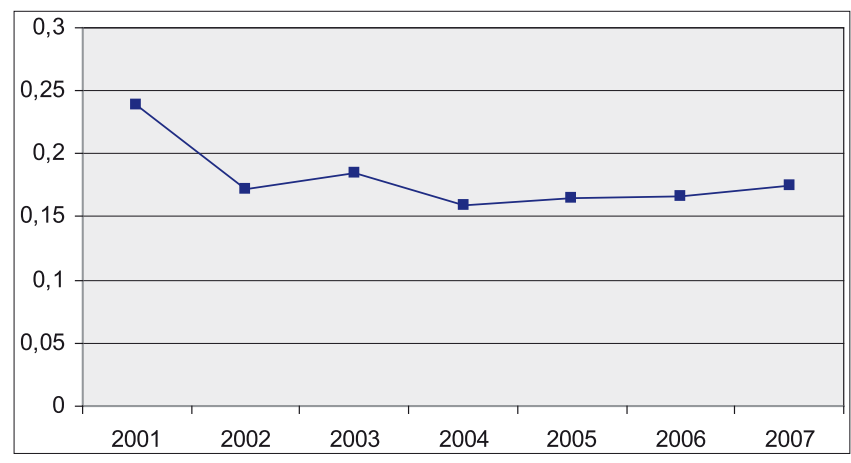

Gráfico 5: Evolución de la proporción de autores sobre el número de suscriptores de la lista de correo electrónico IweTel (2001-2007)

\begin{tabular}{|c|c|c|}
\hline No mensajes & No autores & $\%$ \\
\hline 1 & 1.288 & 45,13 \\
\hline 2 & 493 & 17,27 \\
\hline 3 & 277 & 9,71 \\
\hline 4 & 139 & 4,87 \\
\hline 5 & 108 & 3,78 \\
\hline 6 & 96 & 3,36 \\
\hline 7 & 61 & 2,14 \\
\hline 8 & 50 & 1,75 \\
\hline 9 & 33 & 1,16 \\
\hline 10 & 28 & 0,98 \\
\hline 11 & 24 & 0,84 \\
\hline 12 & 19 & 0,67 \\
\hline 13 & 24 & 0,84 \\
\hline 14 & 13 & 0,46 \\
\hline 15 & 16 & 0,56 \\
\hline 16 & 20 & 0,70 \\
\hline 17 & 13 & 0,46 \\
\hline 18 & 10 & 0,35 \\
\hline 19 & 10 & 0,35 \\
\hline $20-29$ & 50 & 1,75 \\
\hline $30-39$ & 29 & 1,02 \\
\hline $40-49$ & 17 & 0,60 \\
\hline $50-59$ & 8 & 0,28 \\
\hline $60-69$ & 6 & 0,21 \\
\hline $70-79$ & 6 & 0,21 \\
\hline $80-89$ & 3 & 0,11 \\
\hline $90-99$ & 2 & 0,07 \\
\hline $100-199$ & 7 & 0,25 \\
\hline $200-299$ & 2 & 0,07 \\
\hline $300-399$ & 2 & 0,07 \\
\hline
\end{tabular}

Tabla 2. Porcentaje del número de mensajes por autor de la lista de correo electrónico IweTel (2001-2007)
De entre los 549 autores que componen el $20 \%$ de masa crítica de la lista, destacan aquellos que superan los 50 mensajes anuales, entre los que sobresale Tomàs Baiget, creador y director de IweTel (tabla 3).

En cuanto al sexo de los autores, hay un cambio significativo respecto al período 1998-2000 (OntalbaRuipérez, 2002): mientras que entonces había un predominio de mensajes de hombres $(67,7 \%)$, entre 2001 y 2007 las mujeres son responsables del 63\% de la producción de IweTel (gráfico 6).

\begin{tabular}{|c|c|}
\hline Autor & Mensajes \\
\hline Tomàs Baiget & 375 \\
\hline Oskar Calvo & 311 \\
\hline Isidro F. Aguillo & 269 \\
\hline Mariana Pineda & 261 \\
\hline Jesús Tramullas & 134 \\
\hline José Antonio Frías & 129 \\
\hline Francisco Tosete Herranz & 118 \\
\hline Paco López Hernández & 115 \\
\hline Javier Leiva Aguilera & 105 \\
\hline Jorge Serrano Cobos & 105 \\
\hline Albert Blanch & 104 \\
\hline Àngels Massísimo & 96 \\
\hline Hugo Contreras Navarro & 95 \\
\hline Rosa Martínez & 84 \\
\hline Pedro Hípola Ruiz & 83 \\
\hline Cristóbal Pasadas Ureña & 81 \\
\hline José Ramón Pérez Agüera & 77 \\
\hline Alfonso Moreira & 76 \\
\hline Carolina Martín Carretero & 74 \\
\hline Javier Gimeno Perelló & 72 \\
\hline María Pilar Gil García & 72 \\
\hline Blanca Calvo & 70 \\
\hline Alice Keefer & 67 \\
\hline Miguel Benito & 65 \\
\hline José Manuel García Catalán & 64 \\
\hline Montse Monge & 63 \\
\hline Francisco José Diago Márquez & 62 \\
\hline José Raúl Vaquero Pulido & 61 \\
\hline Felipe Meneses Tello & 59 \\
\hline Catuxa Seoane & 57 \\
\hline Rafael Muñoz Muñoz & 57 \\
\hline Roser Lozano Díaz & 57 \\
\hline Asunción Trénor Galindo & 52 \\
\hline Silvia Senz Bueno & 51 \\
\hline Álvaro Roldán López & 50 \\
\hline Jesús Castillo & 50 \\
\hline
\end{tabular}

Tabla 3. Autores más productivos de la lista de correo electrónico IweTel (2001-2007) y número de mensajes que han enviado 


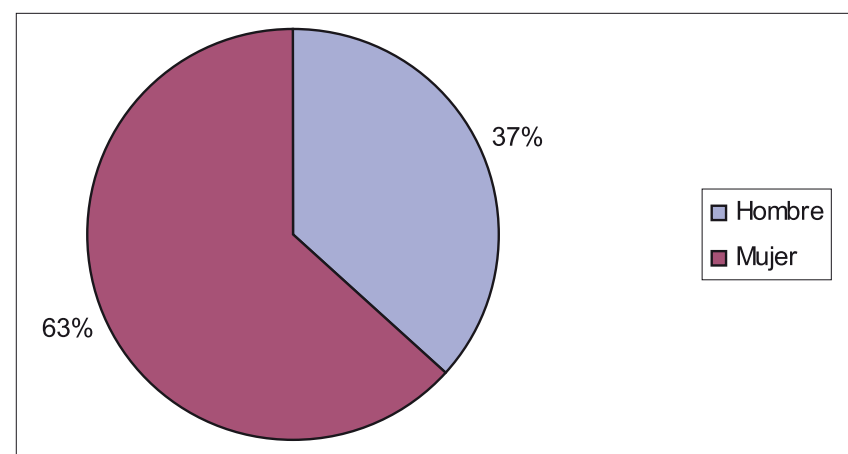

Gráfico 6. Distribución del número de mensajes por sexo de la lista de correo electrónico IweTel (2001-2007)

En cambio, como se observa en el gráfico 7, es mayor el número de autores (53\%) que de autoras (47\%). Es decir, es mayor el número de hombres que publican mensajes que de mujeres, pero ellos envían menos mensajes por persona.

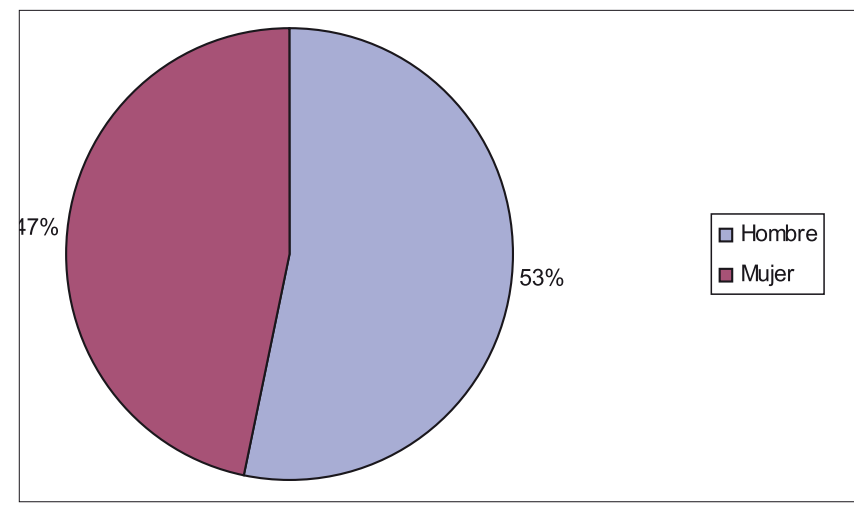

Gráfico 7. Distribución del número de autores por sexo de la lista de correo electrónico IweTel (2001-2007).

A pesar de estas cifras, no deja de ser curioso que, según la tabla 3 , sólo haya una mujer entre los 10 autores más productivos, y cuatro entre los 20 . A partir de ahí la tendencia se va suavizando: nueve mujeres entre los 30 primeros, doce entre los $35 \ldots$ Es decir, y considerando lo anteriormente expuesto, se observa que en la cola de distribución de la autoría de mensajes, las mujeres dominan el tramo medio y los hombres destacan en la cabeza y, sobre todo, en el tramo final, donde muchos de ellos son responsables de unos pocos mensajes.

El origen geográfico por comunidades autónomas (tabla 4) da un previsible predominio de Madrid y, en menor medida, de Cataluña. Destacan la Comunidad Valenciana (por encima, incluso, de Andalucía), y Aragón y Castilla-La Mancha por delante de Murcia o el País Vasco.

Si el origen geográfico se observa desde una escala provincial, y no regional, se advierte que las 8 provincias más productivas (que envían el $80 \%$ de los mensajes de la lista) son, precisamente, aquellas en las que

\begin{tabular}{|l|c|c|}
\hline Comunidad autónoma & Mensajes & $\%$ \\
\hline Comunidad de Madrid & 4.104 & 39,04 \\
\hline Cataluña & 2.451 & 23,32 \\
\hline Comunidad Valenciana & 807 & 7,68 \\
\hline Andalucía & 786 & 7,48 \\
\hline Castilla y León & 469 & 4,46 \\
\hline Aragón & 365 & 3,47 \\
\hline Castilla-La Mancha & 306 & 2,91 \\
\hline Región de Murcia & 276 & 2,63 \\
\hline Galicia & 249 & 2,37 \\
\hline País Vasco & 249 & 2,37 \\
\hline Extremadura & 213 & 2,03 \\
\hline Islas Canarias & 99 & 0,94 \\
\hline Navarra & 43 & 0,41 \\
\hline Asturias & 35 & 0,33 \\
\hline La Rioja & 33 & 0,31 \\
\hline Cantabria & 27 & 0,26 \\
\hline
\end{tabular}

Tabla 4. Distribución de la autoría por comunidades autónomas españolas de la lista de correo electrónico IweTel (2001-2007)

\begin{tabular}{|l|c|r|}
\hline \multicolumn{1}{|c|}{ Lugar procedencia } & Mensajes & \multicolumn{1}{c|}{ \% } \\
\hline Madrid & 4.104 & 39,04 \\
\hline Barcelona & 2.303 & 21,91 \\
\hline Valencia & 684 & 6,51 \\
\hline Zaragoza & 356 & 3,39 \\
\hline Granada & 291 & 2,77 \\
\hline Murcia & 276 & 2,63 \\
\hline Salamanca & 257 & 2,44 \\
\hline La Coruña & 208 & 1,98 \\
\hline
\end{tabular}

Tabla 5. Distribución de la autoría por provincias españolas más productivas en la lista de correo electrónico IweTel (2001-2007)

existen estudios universitarios de Documentación (tabla 5).

Por su parte, la participación internacional en Iwe$\mathrm{Tel}$ la protagoniza Hispanoamérica (con un predominio notable de Argentina), con una presencia menor de Francia, Estados Unidos o Andorra (gráfico 8).

En cuanto a sector, resalta el enorme predominio de la Administración Pública que, incluyendo a la Universidad, abarca casi el $70 \%$ de mensajes enviados a IweTel (gráfico 9); dato nada sorprendente habida cuenta el marcado perfil público del mercado laboral de Documentación (Delgado-López-Cózar y De-la-MonedaCorrochano, 2008).

La tabla 6 confirma el predominio de la autoría universitaria (cerca de la mitad de mensajes) e incluye el único caso de entidad internacional en los primeros puestos (la Universidad Nacional Autónoma de México); destaca, asimismo, la presencia de la empresa y de 


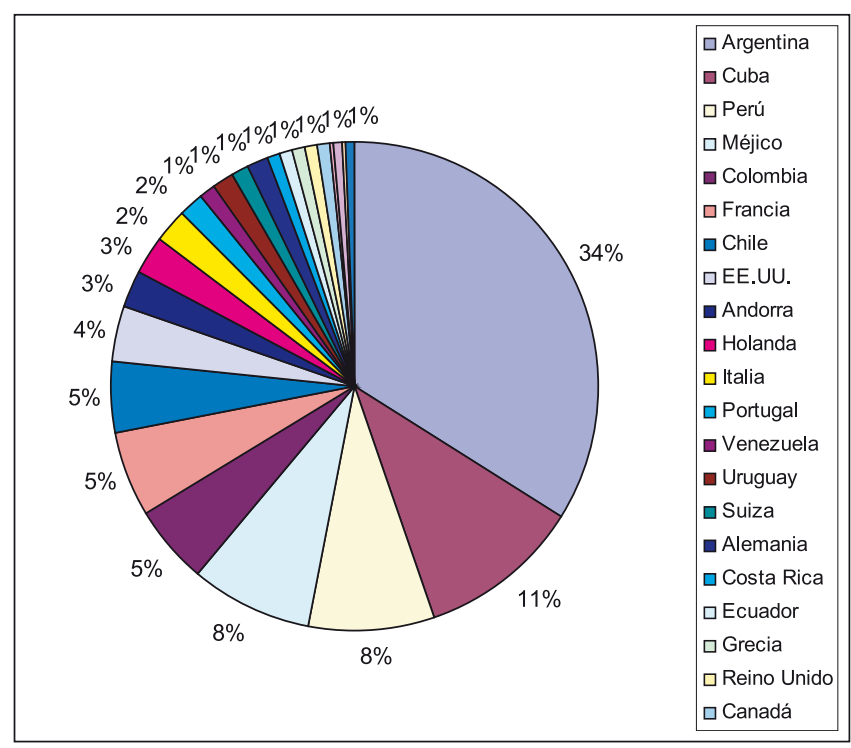

Gráfico 8. Distribución de la autoría por países (excluyendo España) de la lista de correo electrónico IweTel (2001-2007)

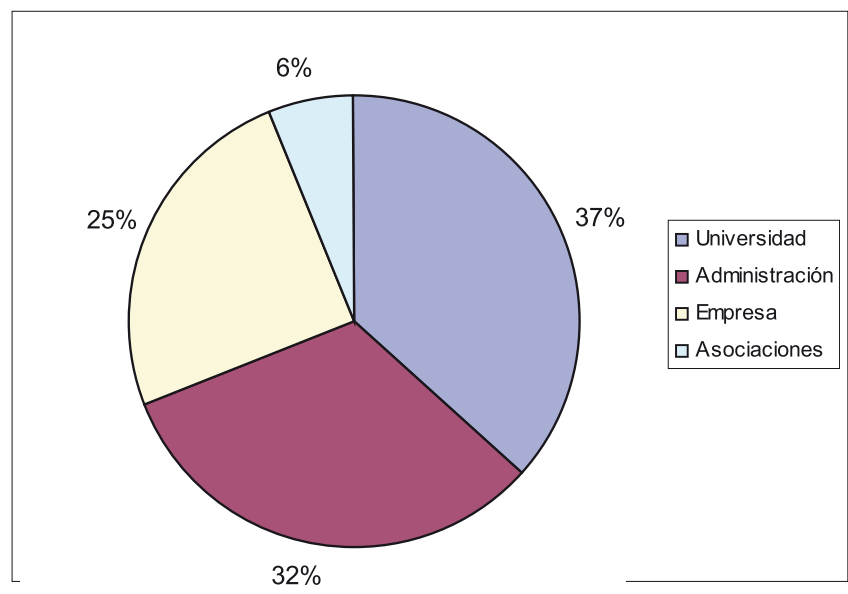

Gráfico 9. Distribución de la tipología de filiación profesional de los autores de la lista de correo electrónico IweTel (2001-2007)

las asociaciones, que incide en el carácter profesional de la lista; finalmente, sorprende el discreto papel de los servicios públicos en estos primeros puestos.

\subsection{Contenido}

\section{Tipología}

En lo referente a los tipos de mensajes, se observa (gráfico 10) un gran número de peticiones de información; a éstas parece corresponder una cantidad aún mayor de respuestas por parte de la lista, aunque la distribución de contestaciones por pregunta es irregular. Alrededor de un 7,2\% de los solicitantes de información redifunde una compilación de las respuestas recibidas, y gracias a estos casos se sabe que muchos miembros contestan las preguntas directamente al interesado, sin enviarlas por la lista.

Se observa (tabla 7) que la media de porcentajes contestados es del 46,5\% de las peticiones de información (con un margen que puede ir de 1 a 29 respuestas

\begin{tabular}{|c|c|}
\hline Institución de procedencia & Mensajes \\
\hline Universidad Complutense de Madrid & 501 \\
\hline Universidad Carlos III & 450 \\
\hline $\begin{array}{l}\text { Idescat (Institut d'Estadística de } \\
\text { Catalunya) }\end{array}$ & 375 \\
\hline Cindoc - CSIC & 366 \\
\hline Universitat de Barcelona & 365 \\
\hline $\begin{array}{l}\text { Sedic (Asociación Española de } \\
\text { Documentación e Información) }\end{array}$ & 323 \\
\hline Universidad de Zaragoza & 315 \\
\hline Baratz & 302 \\
\hline $\begin{array}{l}\text { Cobdc (Col.legi Oficial de Bibliotecaris- } \\
\text { Documentalistes de Catalunya) }\end{array}$ & 281 \\
\hline Universidad de Granada & 270 \\
\hline $\begin{array}{l}\text { Junta de Comunidades de Castilla-La } \\
\text { Mancha }\end{array}$ & 249 \\
\hline Universitat Autònoma de Barcelona & 178 \\
\hline Universidad Politécnica de Valencia & 143 \\
\hline Fundación Germán Sánchez Ruipérez & 137 \\
\hline Universidad de Valencia & 137 \\
\hline $\begin{array}{l}\text { IMED (Instituto Madrileño de Estudios } \\
\text { Documentales) }\end{array}$ & 134 \\
\hline Universidad de Castilla-La Mancha & 130 \\
\hline Ministerio de Cultura & 127 \\
\hline $\begin{array}{l}\text { UNED (Universidad Nacional de Educación } \\
\text { a Distancia) }\end{array}$ & 125 \\
\hline Fundación Ciencias de la Documentación & 124 \\
\hline Comunidad de Madrid & 120 \\
\hline Universidad de Alcalá de Henares & 118 \\
\hline Anabad & 116 \\
\hline Universitat Oberta de Catalunya & 115 \\
\hline excatedra.info & 112 \\
\hline Universidad de Murcia & 111 \\
\hline Universidad Nacional Autónoma de Méjico & 111 \\
\hline Doc6 & 110 \\
\hline MASmedios & 109 \\
\hline Catorze & 105 \\
\hline Docugrupo & 104 \\
\hline Universidad Politécnica de Madrid & 103 \\
\hline Universitat Pompeu Fabra & 101 \\
\hline
\end{tabular}

Tabla 6. Distribución de la autoría por filiación profesional de la lista de correo electrónico IweTel (2001-2007)

por pregunta). En esta línea no hay una tendencia clara, salvo el descenso generalizado en peticiones y contestaciones (gráfico 11), el nivel siempre bajo de difusión de respuestas, y la disminución, en los dos últimos años, de las consultas sin responder. Ello parece denotar una mayor colaboración de los usuarios de la lista a la hora de ayudar a aquellos que lo solicitan, pero una menor disposición a compartir las respuestas recibidas quizás porque éstas les llegan a través de la lista públicamente 


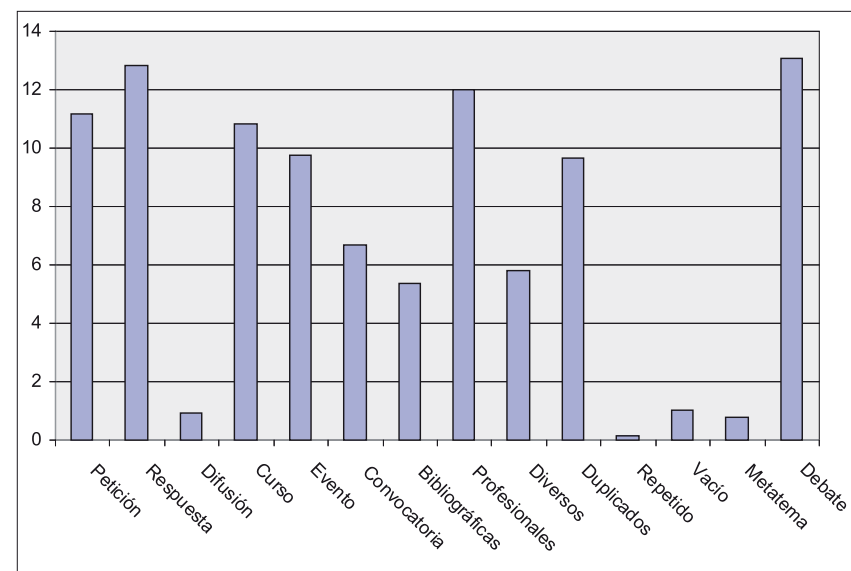

Gráfico 10. Distribución porcentual de las categorías de mensajes de la lista de correo electrónico IweTel (2001-2007)

\begin{tabular}{|l|c|c|}
\hline & \% respondidas & \% difundidas \\
\hline $\mathbf{2 0 0 1}$ & 34 & 6 \\
\hline $\mathbf{2 0 0 2}$ & 29 & 14 \\
\hline $\mathbf{2 0 0 3}$ & 57 & 5 \\
\hline $\mathbf{2 0 0 4}$ & 49 & 8 \\
\hline $\mathbf{2 0 0 5}$ & 40 & 5 \\
\hline $\mathbf{2 0 0 6}$ & 50 & 7 \\
\hline $\mathbf{2 0 0 7}$ & 67 & 6 \\
\hline
\end{tabular}

Tabla 7. Porcentaje de peticiones de información respondidas y de respuestas difundidas (respecto al total de peticiones) en la lista de correo electrónico IweTel (2001-2007)

y consideran que no es necesario volver a remitirlas a IweTel.

Los anuncios de cursos, eventos y convocatorias varían mucho de un año para otro, pero, en conjunto, aumentan respecto del período anterior: los cursos pasan del 6,8 al $11 \%$, los eventos del 8 al $10 \%$, y las convocatorias del 5,3 al 7\% (gráfico 10).

Las novedades bibliográficas permanecen estables, pero las profesionales aumentan su número entre 2001 y 2007 con una media del 5,35\% (gráfico 10). Mientras que los mensajes diversos tienen una dimensión discreta, los de duplicados se incrementan hasta el 5,81\%, provocando una votación en IweTel (febrero de 2002) para decidir si se continuaba permitiendo la remisión de ese tipo de mensajes a la lista ${ }^{2}$.

El efecto beneficioso de la moderación de IweTel, que ya se comprobó a finales del período 1998-2000, se confirma ahora con la casi inexistencia de mensajes repetidos y vacíos $(0,13$ y $1 \%)$, o sobre metatema $(0,78 \%)$, lo que indica un funcionamiento eficiente de la lista (gráfico 10).

Finalmente, los debates pasan a ser la categoría de mayor tamaño. Sin embargo, se muestran irregulares a lo largo de los años: caen entre 2003 y 2005 (a pesar de la polémica de la transposición de la directiva euro-

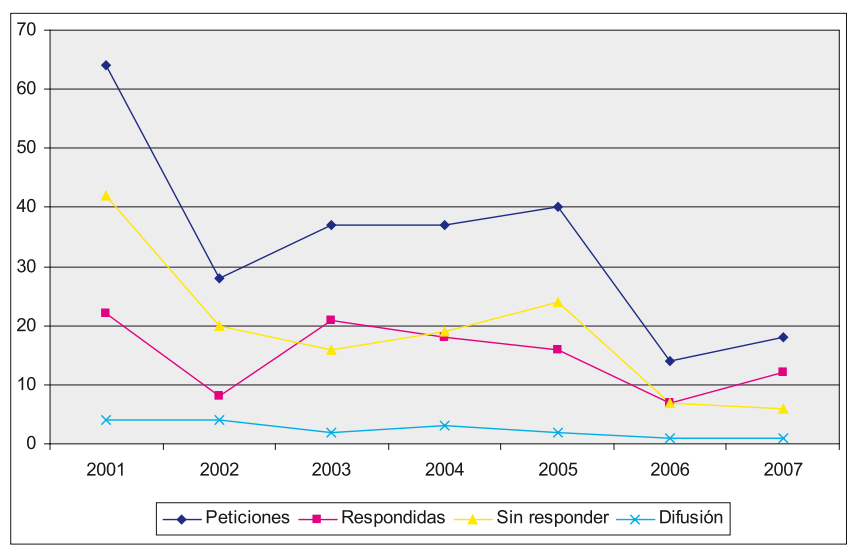

Gráfico 11. Evolución del número de peticiones de información, sus respuestas y difusión de éstas en la lista de correo electrónico IweTel (2001-2007)

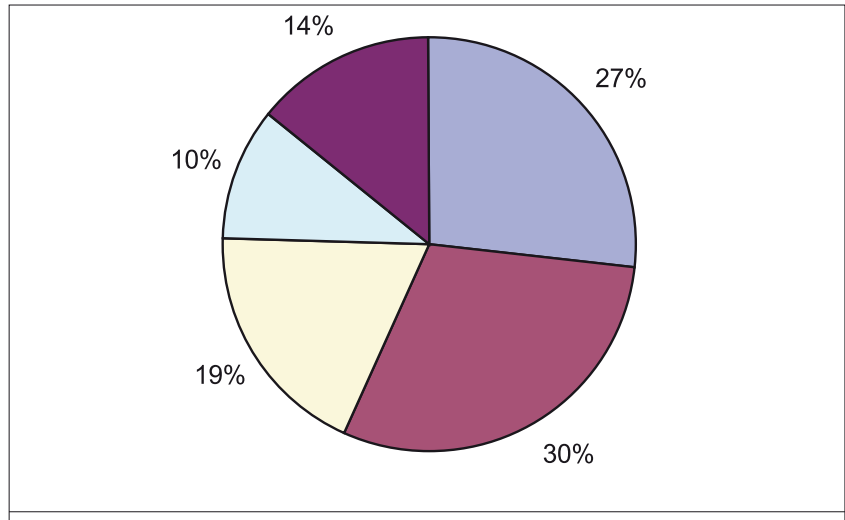

$\square$ Intercambio de inf $\square$ Anuncios $\square$ Novedades $\square$ Duplicados $\square$ Debate

Gráfico 12. Distribución de las agrupaciones de categorías de mensajes de la lista de correo electrónico IweTel (2001-2007)

pea sobre derechos de autor), pero se incrementan en los dos últimos años. En global, y respecto al período 1998-2000, pasan del 8,4 al 13\%.

Valorando ahora los grandes grupos de categorías (gráfico 12), se constata que el uso fundamental de Iwe$\mathrm{Tel}$ es la comunicación profesional, ya que los mensajes de anuncios, novedades y duplicados abarcan el 59\% de este total; le sigue el intercambio de información con un $27 \%$, lo que incide en el papel de la lista como herramienta de creación cooperativa de conocimiento; $\mathrm{y}$, finalmente, los debates (14\%), que muestran a IweTel como foro de discusión de referencia.

\subsection{Temática}

Para identificar los temas más habituales de los debates de la lista, se ha llevado a cabo, por un lado, un vaciado de los debates identificados en la muestra y, por otro, un listado de aquellas discusiones más prolíficas en cuanto a número de respuestas (tabla 8). Para esto último se ha considerado el universo de mensajes, agrupándose en función del título, y seleccionando aquellos que se repetían más (los que seguían un mensaje inicial contestando con un "RE:" en el subject). De esta manera, aparte de los temas que más interés 


\begin{tabular}{|c|c|c|c|}
\hline Título & Autor & Año & Mensajes \\
\hline $\begin{array}{l}\text { El ocaso de las enseñanzas universitarias de Documentación en } \\
\text { España }\end{array}$ & Emilio Delgado & 2007 & 47 \\
\hline Nueva titulación maestro-bibliotecario & Antonia Aleixandre & 2006 & 47 \\
\hline El fin de las Bibliotecas & Belén Álvarez García & 2006 & 32 \\
\hline Licencias de software & Isidro F. Aguillo & 2007 & 32 \\
\hline PROPUESTA: ¿creamos un colegio de documentalistas? & Laura Sánchez & 2007 & 31 \\
\hline Dictadura de los idiotas & Isidro F. Aguillo & 2007 & 29 \\
\hline Biblioteca de Castilleja & Mila Domínguez & 2004 & 27 \\
\hline Alan Story: 10 tesis sobre el sistema internacional del Copyright & Javier Gimeno Perelló & 2007 & 24 \\
\hline Sobre el canon al préstamo en bibliotecas & Hugo Contreras & 2007 & 24 \\
\hline Documentalistas técnicos, un espacio vacío que tenemos que cubrir & Oskar Calvo & 2007 & 21 \\
\hline Algunas de las aplicaciones del P2P & Hugo Contreras & 2007 & 19 \\
\hline Secuestro judicial de una revista & José Vicente Serrano & 2007 & 18 \\
\hline Horror, los estudiantes en la biblioteca & Carolina Martín Carretero & 2003 & 17 \\
\hline Sugerencias sobre Fesabid & Estefanía Aguilar Moreno & 2007 & 17 \\
\hline Perfiles del gestor del conocimiento & Francisco José Bonachera & 2007 & 15 \\
\hline Prohibición de tocar los libros en una biblioteca pública & Lara Campos García & 2006 & 15 \\
\hline Ley de la Lectura, del Libro y de las Bibliotecas & Juan Sánchez Sánchez & 2006 & 14 \\
\hline Recientes fraudes científicos: la punta del iceberg & Emilio Delgado & 2006 & 14 \\
\hline ¿Problemas con los usuarios $2.0 ?$ & Oskar Calvo & 2007 & 13 \\
\hline Debate sobre la neutralidad de la Red & Elvira San Millán & 2006 & 13 \\
\hline Autoempleo & Javier Leiva & 2006 & 12 \\
\hline Los papeles de Salamanca & Mariana Pineda & 2004 & 11 \\
\hline URLs amigables en las webs institucionales. & Oskar Calvo & 2007 & 11 \\
\hline Biblioteca = ¿videoclub? & Arturo Sánchez & 2005 & 10 \\
\hline Autocitas & Tomàs Baiget & 2006 & 10 \\
\hline
\end{tabular}

Tabla 8. Títulos, autores, año y número de mensajes de los debates más prolíficos de la lista de correo electrónico lweTel (2001-2007)

han suscitado, también se han identificado los autores que han conseguido ser más provocadores (teniendo en cuenta que son los que actúan de resorte en los usuarios de la lista llevándoles a implicarse en una discusión); aunque puede darse el caso de que el mensaje inicial no sea más que el reenvío de una noticia de actualidad polémica, con lo que la influencia de ese autor sea discutible.

Por lo que se puede observar, los 25 debates más fructíferos corresponden, básicamente, a los dos últimos años.

A partir de los debates identificados tanto en el total de la población de este estudio (siguiendo los "RE:" en los subjects) como en la muestra, se han considerado los siguientes temas, agrupados en cinco grandes puntos, que se entremezclan a lo largo de las discusiones:

\section{Profesión:}

- Docencia en Documentación: cuál será el futuro de los estudios y su adecuación a las necesidades profesionales.

- Futuro de la profesión/prospectiva: cuestiona- miento sobre la necesidad del profesional de la información para el mercado laboral.

- Intrusismo profesional y precariedad laboral.

- Papel de las asociaciones y los colegios profesionales.

\section{Servicios bibliotecarios:}

- Marketing en las bibliotecas: usuarios o clientes.

- Problemas con usuarios: uso del móvil, accesos a internet, emigrantes, uso de la biblioteca como sala de estudio.

- Nuevos servicios bibliotecarios: desde el Bibliometro de Madrid hasta la Biblioteca 2.0.

- Alfabetización y brecha digital.

\section{Política:}

- Dirección de la Biblioteca Nacional: perfil del cargo.

- Polémicas referentes al Archivo de la guerra civil y a la Ley de la Lectura, del Libro y las Bibliotecas. 
- Problemas de censura: los casos de Google y de El jueves.

- Propiedad intelectual: legislación y canon del préstamo.

- Préstamo en bibliotecas.

- Software libre y su uso por parte de las Administraciones.

\section{Tecnología:}

- Buscadores; modelos de negocio en Red; funcionamiento, valoración y uso de herramientas.

\section{Metatema}

- Idoneidad del envío de mensajes de duplicados a la lista; uso identificativo de los subject de los mensajes.

Respecto a los autores más productivos en los debates (tabla 9), destacan por su presencia en otras tablas (3 y 8): Óskar Calvo, Paco López-Hernández, Tomàs Baiget, Francisco Tosete-Herranz, Mariana Pineda e Isidro Aguillo (que ha perdido algo de presencia en los últimos años).

\begin{tabular}{|l|c|}
\hline \multicolumn{1}{|c|}{ Autor } & Mensajes \\
\hline Óskar Calvo & 12 \\
\hline Paco López Hernández & 10 \\
\hline Francisco José Diago Márquez & 7 \\
\hline Tomàs Baiget & 7 \\
\hline Francisco Tosete Herranz & 6 \\
\hline Mariana Pineda & 6 \\
\hline Javier Gimeno Perelló & 5 \\
\hline José Manuel García Catalán & 5 \\
\hline Pedro López López & 5 \\
\hline Isidro F. Aguillo & 4 \\
\hline Àngels Massísimo & 4 \\
\hline Alejandro Martínez Andaluz & 4 \\
\hline María Jesús del Olmo & 4 \\
\hline María Jesús Tobías Rubio & 4 \\
\hline Angélica Sara Zapatero Lourinho & 3 \\
\hline Antonio Andujar & 3 \\
\hline Carolina Martín Carretero & 3 \\
\hline Chema Pérez & 3 \\
\hline Isabel Andreu Felipe & 3 \\
\hline Isabel Gutiérrez & 3 \\
\hline Jesús Tramullas & 3 \\
\hline José Ramón Pérez Agüera & 3 \\
\hline Roser Lozano Díaz & 3 \\
\hline
\end{tabular}

Tabla 9. Autores más productivos en los debates de la lista de correo electrónico IweTel (2001-2007)

\section{Discusión}

Las razones de la disminución del número de suscriptores en 2006 y 2007 (gráfico 4) probablemente haya que buscarlas en el despegue de vías de comunicación alternativas como las redes sociales o, sobre todo, los blogs. Otra posibilidad puede ser un despegue en la actividad de otras listas como Públicas o Arxifórum que haya provocado el abandono de los suscriptores de IweTel especializados en bibliotecas públicas o archivos: en el caso de Arxifórum, su número de suscriptores ha aumentado en 250 en el período 2005-2008, aunque no necesariamente han de venir desde IweTel; en lo que respecta a Públicas, no hay datos. Finalmente, también habría que considerar el papel de la moderación de la suscripción que se llevó a cabo durante 2006.

La mayor presencia femenina y de mensajes de mujeres en la lista (gráficos 6 y 7) en estos últimos años viene a reflejar con mayor fiabilidad el peso real de la población de mujeres profesionales de la información, que, como indican Delgado-López-Cózar y De-la-Moneda-Corrochano (2008), llega al 70\% de los estudiantes de Documentación.

Como se ha indicado en la tabla 5, parece darse una relación entre el origen geográfico de los mensajes de IweTel y la presencia de estudios de Documentación en una provincia. Salamanca y La Coruña han descendido respecto del período 1998-2000, y cerca del 40\% de los mensajes de Zaragoza han sido enviados por Jesús Tramullas.

En lo referente a la filiación profesional (tabla 6 y gráfico 9), se mantiene (respecto a 1998-2000) la gran presencia de la universidad española, aumenta la representación empresarial (que pasa del 14,6\% al 25\%), consciente del poder de comunicación de la mayor lista de distribución castellanohablante, y aparecen las asociaciones. Para la explicación de esto último, hay que considerar (según Delgado-López-Cózar y De-laMoneda-Corrochano, 2008) el notable incremento de asociaciones profesionales en España durante los últimos años y, con ello, el probable interés de éstas en hacer uso de la lista como vehículo de visibilidad social.

Todo ello, además, debe ponderarse al considerar el peso excesivo de la individualidad en algunos casos. Así, las empresas apenas aparecerían en la tabla 6 de no ser por Jorge Serrano-Cobos, Javier Leiva o Albert Blanch (que envían el 100\% de los mensajes correspondientes a MASmedios, Catorze o Docugrupo, respectivamente). Lo mismo ocurre con la discreta Administración Pública, donde, en realidad destacan los nombres de Tomàs Baiget (100\% de Idescat), Isidro Aguillo o Carolina Martín (de Cindoc y Ministerio de Cultura, respectivamente). Hay que añadir que, en el caso de los servicios públicos, su escasa presencia 
en los primeros puestos (que, como se ha visto, se debe a unos pocos individuos) contrasta con el volumen de mensajes que envía dentro del total de tipologías de filiaciones: un 32\% (gráfico 9), lo que indica su destacada presencia en la "cola de Pareto" de la distribución del conjunto de mensajes de la lista.

En cuanto a la tipología de mensajes, IweTel se reafirma como herramienta de comunicación profesional con el aumento generalizado de mensajes de anuncios, novedades y debates. Sólo se mantiene bajo el número de mensajes de difusión de respuestas, pero ello puede tener su explicación en el destacable porcentaje de consultas que se responden públicamente en la lista (un $67 \%$ en 2007), lo que puede crear la opinión de no que es necesario volver a remitir a IweTel mensajes que ya se han enviado.

En cualquier caso, es notable la mejora de la gestión de la lista, no sólo por la práctica desaparición de mensajes vacíos, repetidos o metatema, sino también por la manera de conducirse los debates, en los que apenas se constata visceralidad (a pesar de que puedan darse posturas fuertemente encontradas) y se observa una mejora de sus contenidos, ganando calidad técnica y profesional.

Y esto último a pesar de que se comprueba que no hay una gran variedad en cuanto a la temáticas generales de los debates respecto al período 1998-2000; eso sí, lógicamente, hay pequeñas variaciones en los temas más específicos debidos a novedades coyunturales. Esto puede significar, entre otras cosas, una renovación de una parte de los autores, que hace que vuelvan a repetirse temas ya tratados años atrás.

A la hora de establecer los autores más influyentes de IweTel no habría que pensar sólo en los más fecundos, ya que se dan no pocos casos de usuarios de la lista que envían sistemáticamente mensajes de anuncios, novedades o duplicados, muchas veces con un eco nulo; esa remisión constante es la que les hace aparecer como prolíficos. Por el contrario, para identificar a los más influyentes habría que cruzar la lista de los más productivos (tabla 3) con la de los que generan más debates (tabla 9) y con la de los iniciadores de los debates más prolíficos (tabla 8).

Considerando lo anterior, los autores que aparecen en los tres casos y que, por tanto, pueden ser considerados con más impacto de IweTel son: Tomàs Baiget, Oskar Calvo, Mariana Pineda, Isidro Aguillo, Javier Gimeno-Perelló y Carolina Martín-Carretero.

Por detrás de éstos habría que tener en cuenta a los que aparecen en dos de las listas. Así, entre los iniciadores de debates más extensos y los más productivos se encuentran: Hugo Contreras y Javier Leiva.

Finalmente, aparecen en las listas de los más productivos (en la de debates y en la general): Àngels Massísimo, Francisco-José Diago-Márquez, Fran-

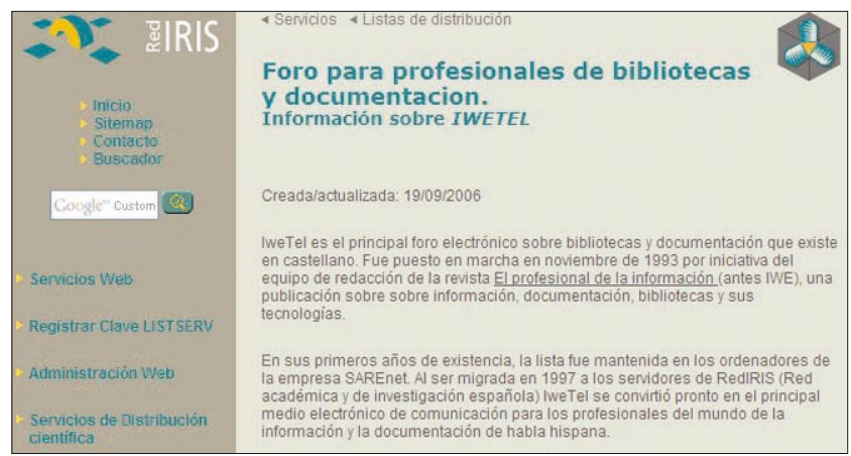

cisco Tosete, Jesús Tramullas, José Manuel GarcíaCatalán, José-Ramón Pérez-Agüera, Paco LópezHernández y Roser Lozano.

Al final del período 1998-2000 se indicó que IweTel había alcanzado una etapa de madurez y que podría en un futuro encaminarse hacia una comunidad virtual. Actualmente, la lista se mantiene en esa etapa de madurez, mejorando la eficiencia de su gestión y mostrándose como una herramienta activa de comunicación profesional que no parece acabar formando parte de una comunidad virtual, pero que tiene que hacer frente a las nuevas formas de comunicación social.

\section{Conclusiones}

1. El número de mensajes enviados a IweTel ha mantenido un crecimiento constante desde 2004, alcanzando los 3.276 en 2007. Sin embargo, el número de suscriptores no ha dejado de descender desde los 5.500 en marzo de 2006 hasta los 4.200 a finales de 2007, con una estabilización a 4.400 a lo largo de 2008.

2. Para el período 2001-2007 se han identificado 2.854 autores (un $17 \%$ de los suscriptores), que han remitido a la lista una media de 6,1 mensajes.

3. El $20 \%$ de los autores envía el $76 \%$ de los mensajes.

4. El $47 \%$ de los autores es mujer y publica el $63 \%$ de los mensajes de la lista, y destaca en el tramo medio de la cola de distribución de éstos.

5. Sobresalen Madrid y Cataluña (seguidas de Comunidad Valenciana, Andalucía y Castilla y León) como las regiones más productoras en IweTel. A nivel internacional, predomina Argentina.

6. El ámbito universitario es el preponderante como origen profesional de los autores, por encima del resto de organismos públicos o privados.

7. El uso fundamental de IweTel es la comunicación profesional (anuncios, novedades, solicitudes de información) y, en menor medida, el foro de discusión (un $14 \%$ frente al $86 \%$ de los anteriores).

8. Los temas más habituales en la lista son: profesión, servicios bibliotecarios, política, tecnología y metatema. 
9. Los autores con mayor impacto son: Tomàs Baiget, Oskar Calvo, Mariana Pineda, Isidro F. Aguillo, Javier Gimeno-Perelló y Carolina MartínCarretero.

\section{Notas}

1. Datos recogidos del archivo de la página: http://listserv.rediris.es/archives/

en Internet Archive:

http://www.archive.org

2. RedIris implementó un filtro topic, de manera que los usuarios pueden configurar su suscripción a IweTel para no recibir los anuncios de duplicados. Para que el sistema funcione es necesario que los mensajes lleven en el asunto "DUPLI:", cosa de la que se encargan que se cumpla los moderadores.

\section{Bibliografía}

Althaus, Scott L. "Computer-mediated communication in the university classroom: An experiment with on-line discussions". Communication education, 1997, July, v. 46, n. 3, pp. 158-174.

Baiget, Tomàs. "La comunidad bibliotecaria y documental de IweTel". Mi biblioteca, 2008, verano, n. 18, pp. 20-24.

Bar-Ilan, Judit. "The 'mad cow disease', Usenet newsgroups and bibliometric laws". Scientometrics, 1997, v. 39, n. 1, pp. 29-55.

Bar-Ilan, Judit; Assouline, Betty. "A content analysis of Pubyac: a preliminary study". Information technology and libraries, 1997, December, v. 16, n. 4, pp. 165-174.

Berge, Zane L.; Collins, Mauri P. "Perceptions of e-moderators about their roles and functions in moderating electronic mailing lists". Distance education: an international journal, 2000, v. 21, n. 1, pp. 81-100.

http://www.emoderators.com/moderators/modsur97.html

Burnett, Gary. "Information exchange in virtual communities: a typology". Information research, 2000, July, v. 5, n. 4. http://informationr.net/ir/5-4/paper82.html

Burton, Paul F. "Electronic mail as an academic discussion forum". Journal of documentation, 1994, June, v. 50, n. 2, pp. 99-110.

Delgado-López-Cózar, Emilio; De-la-Moneda-Corrochano, Mercedes "Las cifras de la profesión documental en España". Anuario ThinkEPI, 2008, pp. 251-268.

Delgado-López-Cózar, Emilio; De-la-Moneda-Corrochano, Mercedes "Las cifras de la enseñanza universitaria en Documentación en España: 2006". El profesional de la información, 2008, julio-agosto, v. 17, n. 4, pp. 422-436.

Ellis, David; Oldridge, Rachel; Vasconcelos, Ana. "Community and virtual community". Annual review of information science and technology, 2004, v. 38, n. 1 , pp. $145-186$.

Feliu, Víctor. “Com avaluar les llistes de distribució?". Métodos de infor mación, 1999, julio, v. 6, n. 31, pp. 41-52.

García-Testal, Cristina. "Entrevista a Pedro Hípola". Métodos de informa ción, 1999, julio, v. 6, n. 31, julio, pp. 36-39.

Gilas, Tom; Schein, Moshe; Frykberg, Eric. "A surgical Internet discussion list (Surginet) - A novel venue for international communication among surgeons". Archives of surgery, 1998, October, v. 133, n. 10, pp. 1.126-1. 130.

Hartland, Dave. "The networked information-services project (NISP-II) - Bringing the benefits of the computer network to a wider academy community". University computing, 1992, v. 14, n. 3, pp. 85-89.

Hernández-Borges, Ángel A.; Pareras, Luis G.; Jiménez, Alejandro. "Comparative analysis of pediatric mailing lists on the internet". Pediatrics, 1997, August, v. 100, n. 2, pp. e8

Jiménez, José-Antonio; Padilla, Antonio. "Análisis estratégico de la integración de las listas de distribución en las Comunidades Virtuales de Usua- rios”. Boletín de la RedIris, 1998, n. 46-47, pp. 107-114.

Klingler, Scott L. "Online Ph. D. programs: a look at a Jesse listserv discussion thread". Journal of education for library \& information science, 2007, v. 48 , n. 1 , pp. $5-12$

Kuperman, Victor. "Productivity in the internet mailing lists: a bibliometric analysis". Journal of the American Society for Information Science and Technology, 2006, January, v. 57, n. 1, pp.51-59.

Mac Lennan, Birdie. "Serialst and the global serials community: the five year evolution of an electronic discussion forum". Serials review, 1996, v. 22 , n. 3, pp. 1-21.

Merlo-Vega, José-Antonio; Sorli-Rojo, Ángela. "Las relaciones interprofesionales en las listas de distribución de información y documentación españolas". En: VI Jornadas Españolas de Documentación, 1998, Valencia, pp. 597-612.

Merlo-Vega, José-Antonio; Sorli-Rojo, Ángela. "Las listas de distribución como herramienta profesional". Métodos de información, 1999, julio, v. 6, n. 31 , pp. $53-60$

Moreale, Emanuela; Watt, Stuart. "Organisational information management and knowledge discovery in email within mailing lists". Lecture Notes in Computer Science, 2002, v. 2412, pp. 87-92.

Nagel, Kat. The natural life cycle of mailing lists, 1994.

http://www.rider.edu/ suler/psycyber/lifelist.html

Versión española: Ciclo natural de las listas de distribución, 1994.

http://the-geek.org/escepticos/199902/msg00667.html

"New listserv for users of OCLC's EPIC service". Information today, 1994, September, v. 11 , n. 8, p. 39.

Ontalba-Ruipérez, José-Antonio. "Análisis de contenido de la lista de distribución IweTel (1998-2000)". El profesional de la información, 2002, enero-febrero, v. 11, n. 1, pp. 14-27.

Pedersen, Robert C. "A quantitative approach to the description of internet mailing lists". The serials librarian, 1996, v. 30, n. 1, pp. 39-47.

Ray, Eric J. "Techwr-1: a history and case study of a profession-specific listserv list”. Technical communication, 1996, Nov., v. 43, n. 4, pp. 334-338. http://www.raycomm.com/techwhirlcasestudy.html

Robinson, Kara L. "People talking to people: making the most of internet discussion groups". Online, 1996, v. 20, n.1, pp. 26-32.

Rodríguez-Recio, Francisco-Javier; Sendra-Portero, Francisco. "Analysis of the Spanish-speaking mailing list Radiologia". European journal of radiology, 2007, July, v. 63, n. 1, pp. 136-143.

Schoch, Natalie A.; Shooshan, Sonya E. "Communication on a listserv for health information professionals. Uses and users of Medlib-L". Bulletin of the Medical Library Association, 1997, January, v. 85, n. 1, pp. 23-32.

Serrano-Castro, Pedro J.; Arjona-Padillo, Antonio; Guardado-Santervás, Pedro L. "Análisis descriptivo de la lista de correo electrónico 'Neurología-RedIRIS'

http://listserv.rediris.es/neurologia.html

un instrumento activo, plural y de calidad científica al alcance de la neurología hispanohablante". Neurología, 2004, octubre, v. 19, n. 8, pp. 420-428.

Tichon, Jennifer G.; Shapiro, Margaret. "The process of sharing social support in cyberspace". Cyberpsychology \& behavior, 2003, April, v. 6, n. 2, pp. 161-170.

Wildemuth, Barbara M.; Crenshaw, Lisa; Jenniches, William; Harmes, J. Christine. "What's everybody talking about? Message functions and topics on electronic lists and newsgroups in information and library science". Journal of education for library and information science, 1997, Spring, v. 38 , n. 2, pp. 137-156.

José-Antonio Ontalba-Ruipérez, Instituto de Diseño para la Fabricación y Producción Automatizada, Universidad Politécnica de Valencia, Camino de Vera $s / n$, 46022 Valencia.

joonrui@upv.es 
